[Postprint]

Journal of Proteomics 114(1): 71-82 (2015)

1 Schistosome infections induce significant changes in the host biliary proteome.

2

3 Eduardo de la Torre-Escudero, Ricardo Pérez-Sánchez, Raúl Manzano-Román, Ana

4 Oleaga*

5

6

7 Parasitology Laboratory, Instituto de Recursos Naturales y Agrobiología de Salamanca

8 (IRNASA, CSIC). Cordel de Merinas, 40-52, 37008 Salamanca, Spain.

9

$10{ }^{*}$ Corresponding author:

11 Dr. Ana Oleaga

12 Parasitology Laboratory.

13 Instituto de Recursos Naturales y Agrobiología (IRNASA, CSIC)

14 Cordel de Merinas, 40-52

1537008 Salamanca, Spain

16

17 Tel.: +34 923219606; fax: +34923219609

18

19 E-mail address: ana.oleaga@irnasa.csic.es

20 
21 Abstract

22

Schistosomiasis is a disease caused by blood trematodes affecting man and

23 animals that represents an important human health and veterinary problem. Main

24 damages caused by this infection are a consequence of the host inflammatory reaction

25 against the parasite eggs trapped inside the liver. Despite the hepatic pathology of

26 schistosomiasis is very well known, there is no specific studies dealing with the

27 schistosome infection effects on the biliary function. The purpose of this work was to

28 analyse the changes induced by Schistosoma bovis infection in the biliary proteome. For

29 this, whole gallbladders from S. bovis-infected and non-infected mice were dissected,

30 homogenized and fractionated by differential centrifugation. The resulting protein

31 fractions were resolved by SDS-PAGE, the gels were sliced, and the gel pieces analyzed

32 by LCAMS/MS. Altogether, we identified 1,937 proteins, which were classified

33 according to their "protein class" and "molecular function", and then subjected to an

34 "Enrichment analysis". The differences found in gallbladder proteomes between S.

35 bovis-infected and non-infected mice are analysed. We show that chronic schistosome

36 infections cause significant changes in the biliary proteome that may produce

37 physiological alterations and affect the therapeutic actions of drugs when administered

38 to human patients and animals with schistosomiasis.

39

40

41 Key words: Schistosoma bovis, gallbladder, proteome.

42

43 
Schistosomiasis is a severe parasitic disease affecting humans and animals in

47 many tropical and subtropical areas of the world that is caused by flukes of the genus

48 Schistosoma. Schistosoma bovis is a blood-dwelling fluke of ruminants that is 49 genetically and immunologically analogous to the important human pathogen 50 Schistosoma haematobium [1]. Like human schistosomes, S. bovis cercariae penetrate 51 the host body through the skin, reaching the skin's capillary vessels. Subsequently, they 52 migrate through the blood stream towards the lungs, where remain for several days as 53 schistosomulum larvae. Following this, the schistosomula travel to the portal vein and 54 mesenteric vessels, where they finally develop into the adult stage [2]. At this location, 55 the adult schistosome worms release large numbers of eggs, many of which pass 56 through the gut wall to the gut lumen and then exit the host with faeces. However, a

57 significant fraction (up to $50 \%$ ) of the eggs do not pass through the intestinal mucosa 58 towards the lumen and are carried by the portal vein blood flow mainly toward the liver 59 sinusoids, where they remain trapped and induce granulomatous lesions and strong 60 immune responses, which typically result in portal hypertension and hepatic fibrosis [3]. 61 The murine model of schistosomiasis is characterised by portal fibrosis, with 62 limited to absent injury of hepatic parenchyma, significant vascular damage, and an 63 apparently normal liver function $[4,5]$. Recently, it has been reported that during the 64 chronic phase of schistosome infection in mice, the proteins associated with several 65 liver metabolic functions (i.e., the citric acid cycle, the fatty acid cycle and the urea 66 cycle) are markedly downregulated, while the proteins associated with stress responses, 67 acute phase reactants and structural components are all significantly upregulated $[6,7]$. 
In addition, important alterations in the histology of the bile ducts in response to

69 entrapped eggs, including the formation of massive granulomas, have been reported, although neither the underlying pathomechanisms of these hepatic lesions nor the likely

71 changes in bile composition as consequence of such hepatic lesions have been studied $72[8,9]$

Bile is a biological fluid with a complex composition (bile salts, phospholipids, 74 cholesterol, bilirubin, inorganic salts and proteins) that is produced by hepatocytes and 75 transferred through the intrahepatic bile ducts to the gallbladder, where it is stored and concentrated at an approximately ten-fold concentration. In response to meals, bile is 77 released into the duodenum through the common bile duct $[10,11]$. Bile serves diverse 78 functions, ranging from metabolism to transport; it essentially helps to emulsify fats, 79 facilitating their digestion and absorption and as an excretion route for xenobiotics (e.g. 80 toxins and drugs) and endobiotics (e.g. cholesterol and bilirubin) [11]. Since bile is produced in the liver, it can be speculated that the pathological

82 changes induced in the liver and the biliary system during schistosome infection would 83 be reflected as alterations in bile protein composition, and therefore in bile function. In 84 spite of this and its foreseeable physiological impact, there is no information concerning 85 the changes that schistosomal hepatopathy may elicit in the composition of bile.

86 Accordingly, and bearing in mind the crucial functions of bile, in the present

87 work we were prompted to investigate whether the S. bovis infection induces changes in 88 the biliary proteome. To accomplish this, we applied a protocol aimed at reducing the 89 complexity of bile samples, thereby avoiding the presence of molecules that interfere 90 with protein analysis, such as bile salts and lipids [10]. For this, whole gallbladders 91 from S. bovis-infected and non-infected mice were dissected, homogenized and 92 fractionated by differential centrifugation. The resulting protein fractions were resolved 
93 by SDS-PAGE, the gels were sliced, and the gel pieces analyzed by LCAS/MS.

94 Altogether, in infected and non-infected mice we identified 1,937 proteins, which were

95 classified according to their "protein class" and "molecular function", and then

96 subjected to an "Enrichment analysis". The differences found in gallbladder proteomes

97 between S. bovis-infected and non-infected mice are discussed.

98

99 2. MATERIALS AND METHODS.

100

101 2.1. Experimental animals and infection

102

Forty NMRI mice weighing 30-35 g (Harlan Laboratories Models, S.L.) were

103 used. These mice were distributed into two experimental groups of 20 mice per group:

104 G0 group of non-infected mice and G1 group of mice infected with S. bovis.

105

Mouse infection with S. bovis was done by tail immersion with 200 S. bovis

106

cercariae for 60 min. Four months later, when the infections were fully established, all

107 mice were autopsied and their gallbladders extracted and stored at $-80{ }^{\circ} \mathrm{C}$ until

108 processing.

109 All animal manipulations were done according to the rules from the ethical and

110 animal welfare Committee from the Institution where the experiments were conducted

111 (IRNASA, CSIC), following the corresponding EU rules and regulations.

112

113 2.2. Gallbladder protein extracts for proteomic analyses

114 The mouse gallbladders were collected and grouped in batches of 5

115 gallbladders/batch and processed for the preparation of protein extracts. Each batch was

116 resuspended in PBS $(\mathrm{pH}=7.4)$ containing a cocktail of proteinase inhibitors (Roche

117 Diagnostics) (PBS -1 ) and homogenized six times (2 min each) using an Ultra-Turrax 
118 T10 disperser (IKA-Werke) at full power. The homogenates were sonicated for 3 min,

119 and then subjected to a fractionation process by centrifugation to reduce the complexity

120 of the protein composition of samples and facilitate their proteomic analysis. First, the

121 homogenates were centrifuged at $17,000 \times \mathrm{xg}$ and $4^{\circ} \mathrm{C}$ for $30 \mathrm{~min}$ and the resulting

122 supernatants $(S-1)$ and pellets $(P-1)$ were recovered. Next, a fraction of the S-1

123 supernatants was centrifuged at $100,000 \times g$ and $4^{\circ} \mathrm{C}$ for 60 min and the new

124 supernatants (S-2) and pellets ( $P-2$ ) were recovered. The pellets obtained in each 125 centrifugation step were resuspended in $100 \mu$ of PBS - . The protein concentration of

126 all the fractions (supernatants and pellets) was assessed using the BCA Protein Assay 127 Reagent kit (Pierce).

128 Samples of $15 \mu \mathrm{g}$ of each fraction (S-1, S-2, P-1 and P-2) were mixed with $4 \times$ 129 Laemmli buffer [12], heated to $90^{\circ} \mathrm{C}$ for $3 \mathrm{~min}$ and centrifuged at 12,000 $\mathrm{xg}$ for $4 \mathrm{~min}$.

130 The protein samples were then resolved by SDS-PAGE in 10\% polyacrylamide gels and 131 stained either with Coomassie Blue (Coomassie Blue R-25 0.125\%, methanol 50\%, 132 acetic acid 10\%) or silver stain, using the PlusOne Silver Staining Kit (GE Healthcare).

133 The silver-stained gels were digitalized with the ChemiDoc system (Bio-Rad)

134 and gel images were analysed with Image Lab software (Bio-Rad). The gel lanes 135 stained with Coomassie blue were sliced into 3 pieces and these slices were sent to the 136 Proteomic Service of the University of Valencia (Spain) for protein identification.

138 2.3. In-gel enzymatic digestion and liquid chromatography and tandem mass 139 spectrometry (LC-MS/MS).

140 For each $\mathrm{G} 0$ and $\mathrm{G} 1$ group, two independent batches of five gallbladders each 141 were subjected to enzymatic digestion and LCAMSMS analysis. For all the samples,

142 technical replicas were obtained by injecting each sample twice. 
144 with sequencing grade trypsin (Promega) $\left(20 \mathrm{ng} / \mu \mathrm{l}\right.$ in $\left.25 \mathrm{mM} \mathrm{NH}_{4} \mathrm{HCO}_{3}\right)$ overnight at $14537^{\circ} \mathrm{C}$. The reactions were stopped with $10 \%$ trifluoroacetic acid (TFA) at a final 146 concentration of $0.1 \%$, and the supernatants were filtered through a $0.22 \mu \mathrm{m}$ filter and 147 dried by centrifugation in a vacuum. The concentration of peptides was estimated by 148 UV spectrometry, assuming that a $1 \mathrm{mg} / \mathrm{ml}$ solution of proteins had an extinction 149 coefficient of 1.1 absorbance units at $280 \mathrm{~nm}$.

$150 \quad$ The peptides extracted following in-gel digestion were resuspended in $6 \mu$ of $1515 \%$ acetonitrile, $0.1 \%$ TFA, and $5 \mu$ of the sample was loaded onto a trap column 152 (NanoLC Column, $3 \mu \mathrm{C} 18-\mathrm{CL}, 350 \mu \mathrm{m} \times 0.5 \mathrm{~mm}$, Eksigen) and desalted with $0.1 \%$ TFA 153 at a flow rate of $3 \mu \mathrm{l} / \mathrm{min}$ for $5 \mathrm{~min}$. The peptides were then loaded onto an analytical 154 column (LC Column, $3 \mu \mathrm{C} 18-\mathrm{CL}, 75 \mu \mathrm{m} \times 12 \mathrm{~cm}$, Nikkyo) equilibrated in 5\% acetonitrile and $0.1 \%$ formic acid. The peptides eluted were analyzed with a nanoESIQ-TOF mass spectrometer (5600 TripleTOF, ABSciex) in information-dependent 157 acquisition mode, in which a 0.25 -s TOF MS scan from 350 to $1250 \mathrm{~m} / \mathrm{z}$ was 158 performed, followed by $0.05-s$ product ion scans from 100 to $1500 \mathrm{~m} / \mathrm{z}$ on the 50 most 159 intense 2-5 charged ions.

2.4. Database searching and protein identification Database searches were performed in the NCBInr Mus musculus database $(35,149,712$ sequences; $12,374,887,350$ residues) using the Mascot v2.2 (Matrix

164 Science) search engine. For the samples from the Gl group, an additional search was 165 performed in a custom-made Trematoda_EST database $(2,472,666$ sequences; $396,860,374$ residues) using the nucleotide sequences published in the NCBI_EST 
167 database. This search was performed to evaluate the potential presence of proteins of 168 parasite origin.

169 For the Mascot searches, the peak lists were generated directly from QSTAR 170 wiff files by Mascot Daemon v. 2.2.2 (Matrix Science) with Sciex Analyst import filter

171 options using the default parameters. Databases were searched using the following 172 parameters: tryptic specificity, allowing one missed cleavage and a tolerance on the 173 mass measurement of 70 ppm in MS mode and 0.6 Da for MS/MS ions. The 174 carbamidomethylation of Cys was set as a fixed modification, and Met oxidation and 175 Asn/GIn deamidation were set as variable modifications. The significance threshold was 176 set at 0.05 and proteins required at least two unique significant peptides to be 177 considered identified.

178 Redundant identifications were eliminated and the keratins and 179 immunoglobulins found were excluded from the lists of identified proteins provided in 180 the Results section.

181

182 2.5. Data analysis

183 Protein classification was performed according to the Gene Ontology (GO) 184 hierarchy using the Universal Protein Resource (UniProt) retrieval system 185 (http://Www.uniprot.org/). The "ID mapping" module for the UniProt was used to 186 transform the $\mathrm{Gl}$ number to UniProt code, standardize proteins symbols, and associate 187 them with corresponding gene names, gene ontology categories and IDs, molecular 188 functions, subcellular location and tissue specificity. 
190 Panther classification system (http://www.pantherdb.org/) [13] and the "Enrichment

191 Analysis" module of the WEB-based GEne SeT AnaLysis Toolkit (WebGestalt)

192 (http://5ht-dev.accre.vanderbilt.edu/webgestalt) [14]. Enrichment analyses for the Gene

193 Ontology categories (biological process and molecular function) and for the biological

194 pathways in the Wikipathways database were carried out using the mmusculus_genome

195 database as reference set, the hypergeometric test as a statistical method, and $\mathrm{BH}$ as a

196 multiple test adjustment method [15]. The significant level for the GO enrichment

197 analysis for biological process and molecular function was the top 10 categories, and for

198 the Wikipathways enrichment analysis a significance of $p<0.0001$ and a minimum

199 number of 6 genes for each category were selected.

200

201

3. RESULTS

202

203

3.1. Gallbladder protein extracts.

205

Gallbladders from the $\mathrm{G} 0$ and $\mathrm{G} 1$ mice were homogenized in batches of 5 and these homogenates were fractionated by means of two consecutive centrifugations at 17,000 and $100,000 \times g$, respectively. Thus, we obtained 4 fractions: supernatants S-1 and $\mathrm{S}-2$ and pellets $\mathrm{P}-1$ and $\mathrm{P}-2$. The proteins in each of these fractions were resolved by SDS-PAGE and stained with silver for protein visualization.

Fig. 1 shows the results of this fractionation procedure, showing that the $S-1$ and P-1 fractions had complex band patterns that covered a broad range of molecular sizes, and also revealing some differences in protein composition between the $\mathrm{G} 0$ and $\mathrm{Gl}$

212 mouse groups localized around 52 kDa for S-1 and between 34 and 42 kDa for P-1. 
213 Regarding fractions S-2 and P-2, their band patterns were less complex, although they

214 still showed differences between the G0 and G1 mice (Fig. 1).

215 After fractionation, for each fraction (S-1, P-1, S-2 and P-2) all batches of

216 gallbladders from the same mouse group ( $G 0$ or $G 1$ ) showed identical band patterns to

217 that of the homologous fraction in Fig. 1 (not shown), demonstrating the reproducibility 218 of the procedure.

219

220

3.2. Proteins identified.

221

The results presented here are the sum of the identifications performed with

222 Mascot in the NCBInr_Mus musculus database in the two gallbladder batches (5

223 gallbladder/batch) analyzed from each group of mice (G0 and G1). In order to simplify

224 the comparative analysis between the $\mathrm{G} 0$ and $\mathrm{Gl}$ mice, these results do not include keratins, immunoglobulins or immunoglobulin-like proteins.

226 The proteomic analysis of all the gallbladder fractions -supernatants and pellets-

227 allowed the identification, on the basis of at least two significant peptides, of a total of 228 1,937 non-redundant proteins: 1,589 in the gallbladder of non-infected mice (G0) and

2291,489 in the gallbladder of mice infected with S. bovis (G1). Of these proteins, 348 were 230 identified only in the gallbladder of the G1 group; 448 only in the G0 group, and 1141 231 in both groups of mice (Table 1 and Supplementary Table 1). The number of non-redundant proteins identified in individual fractions ranged

233 between 300 and 1069, the S-1 fractions showing the highest values, followed by the P234 1, P-2 and S-2 fractions. The only exception was the P-2 fraction from the G0 group, in which up to 1,044 non-redundant proteins were identified (Table 1). 
subsequent BLAST analysis of these sequences allowed us to identify 154 proteins and

23935 hypothetical, unknown or non-characterized proteins (Supplementary Table 2). All

240 these identified proteins, most of them belonging to highly conserved protein families

241 such as actins, tubulins, heat shock proteins, histones, etc., had already been identified

242 in the NCBInr Mus musculus database, so that any of them could be considered of

243 parasite origin.

244 The proteins identified in all the fractions from the same experimental group of

245 mice, either G0 or G1, were grouped and analyzed together. The proteins were

246 classified first by their "Protein Class" and "Molecular Function" using the Panther

247 Classification System, followed by an "Enrichment Analysis" with the Mus musculus

248 genome as the reference set.

249 Protein classification.

250

The "Protein Class" classification of the biliary proteomes from S. bovis infected

251 and non-infected mice is shown in Fig. 2. For both groups of mice, G0 and G1, the

252 proteins were grouped in the same 28 protein classes, showing a very similar

253 distribution. The most numerous protein classes were oxidoreductases (17.6\% in G0 and

$25415.7 \%$ in $\mathrm{G} 1$ ) and hydrolases (15.1\% in $\mathrm{G} 0$ and $17.6 \%$ in $\mathrm{G} 1$ ), followed by transferases

255 (13.1\% in G0 and 11.8\% in G1), nucleic acid-binding proteins (11.5\% in G0 and 10.8\%

256 in $\mathrm{G} 1$ ), enzyme modulators (7.3\% in $\mathrm{G} 0$ and $8.8 \%$ in $\mathrm{G} 1$ ), cytoskeletal proteins $(9.0 \%$ in

$257 \mathrm{G} 0$ and $8.6 \%$ in $\mathrm{Gl}$ ) and proteases (5.7\% in $\mathrm{G} 0$ and $6.9 \%$ in $\mathrm{G} 1$ ). There were few

258 differences in the protein class ratios between the $G 0$ and $\mathrm{Gl}$ mice, being

259 oxidoreductases and transferases slightly more numerous in the non-infected mice (G0),

260 while hydrolases, proteases and enzyme modulators were slightly more enriched in the

261 infected mice (G1) 

identified in both groups revealed certain particularities, detailed below. In this "Protein

264 Class" 215 non-redundant proteins were included, of which 197 were identified in the

265 G0 group and 174 in G1. In a comparative analysis of the proteins included in the

266 "oxidoreductase proteins class" identified exclusively in each group, 41 in G0 and 18 in

267 G1, the following were observed: (i) In both groups, $G 0$ and $G 1$, proteins involved in 268 different metabolic processes (carbohydrate metabolism, glycogen metabolism, 269 glycolysis, etc) were identified; (ii) 6 of the 7 proteins involved in immune response/inflammation/stress response, were identified only in the Gl group; (iii)

271 proteins involved in lipid metabolism were only identified in the G0 group, and (iv) 8 272 of the 9 components involved in the respiratory electron transport chain, among which 273 several members of the Cytochrome P450 family were included, were identified only in 274 the samples from the G0 group (Supplementary Table 3).

276 gallbladders of groups $\mathrm{G} 0$ and $\mathrm{G} 1$, of the 39 proteins of this family identified jointly in 277 both groups, all were identified in the G0 group but only 16 were present in the 278 gallbladders of the infected mice (G1) (see Supplementary Table 1). The additional "molecular function" classification of the identified proteins resulted in the following categories: catalytic, binding, structural, antioxidant, enzyme 281 regulator, receptor, translation regulator, and transporter activity. In both groups of 282 mice, $\mathrm{G} 0$ and $\mathrm{G} 1$, the most numerous categories were those involving catalytic (49.1\% 283 in $\mathrm{G} 0$ and $49.7 \%$ in $\mathrm{G} 1$ ), binding (21.3\% in $\mathrm{G} 0$ and $21.4 \%$ in $\mathrm{G} 1$ ) and structural activity 284 (11.7\% in G0 and 10.9\% in G1). The remaining categories showed remarkably lower ratios, ranging between $0.8 \%$ and $5.8 \%$ in the $\mathrm{G} 0$ mice and $0.7 \%$ and $5.5 \%$ in the $\mathrm{G} 1$ 
mice; in both mouse groups these ratios corresponded to proteins with antioxidant and transporter activity, respectively. mice, and in both groups of mice (G0 and $G 1)$, classified according to their molecular

290 function are summarized in Fig. 3. Proteins with catalytic function represented 46.3\%,

$29150.0 \%$ and $49.6 \%$ of the identified proteins only in G0, only in G1, and in both groups, respectively. Thus, to avoid masking the relative abundance of the other groups of proteins represented, the catalytic activity category is not included in Fig 3. This figure shows no differences between $\mathrm{Gl}$ and $\mathrm{G} 0$ groups in the percentage of proteins with binding, antioxidant, receptor and transporter activity. By contrast, some differences were observed between both groups of animals in the percentages of proteins with structural (13.3\% in $\mathrm{G} 0$ versus $7.9 \%$ in $\mathrm{G} 1$ ) and translation regulator activity $(4.7 \%$ in $\mathrm{G} 0$ versus $0.8 \%$ in $\mathrm{G} 1$ ), which were both more numerous in $\mathrm{G} 0$ than in $\mathrm{G} 1$. Conversely, proteins with enzyme regulator activity were more numerous in the $\mathrm{G} 1$ group (8\%) than in the $\mathrm{G} 0$ group (1.9\%) (Fig. 3).

Enrichment analysis of the proteins identified. enriched biological pathways, biological processes and molecular functions associated with the bile proteins identified in $\mathrm{G} 0$ and $\mathrm{G} 1$ groups. a significance of $p<0.0001$ and a minimum number of 6 genes for each category. Up to 39 of these 48 pathways proved to be significantly enriched in the gallbladder proteomes of both groups of mice. For some of these 39 pathways, differences in their enrichment ratios were observed between the mouse groups, being typically higher in the G0 than in the G1 mice, and especially evident in the following pathways: oestrogen 
311 metabolism (29.74\% in G0 versus $16.33 \%$ in G1), glucuronidation $(29.34 \%$ in G0

312 versus $22.46 \%$ in $\mathrm{G} 1$ ), mitochondrial beta-oxidation (41.98\% in G0 versus $35.32 \%$ in

$313 \mathrm{G} 1$ ) and oxidative stress (20.28\% in G0 versus $13.61 \%$ in G1) (Fig. 4). circadian exercise, irinotecan pathway, retinol metabolism and TGF-beta receptor signalling pathway proved to be significantly enriched only in the G0 group, and the

317 EGFR1 signalling pathway, the FAS pathway, the IL-6 signalling pathway and $T$ Cell receptor signalling pathway were only enriched significantly in the G1 group.

Table 2 shows the proteins involved in a representative group of enriched pathways, e.g. the oestrogen metabolism, the irinotecan metabolism, the oxidative stress

321 and the TGF-beta receptor signalling pathways. As can be seen, some protein families,

322 such as the UDP-glucuronyl transferases, cytochrome P450, MAP kinase and NAD(P)H 323 dehydrogenase, were involved in several biological pathways. The proportion of 324 proteins identified in the G0 group with respect to those identified in G1, was always 325 higher in the former. Accordingly, this proportion ( $n^{0}$ of proteins identified in the $\mathrm{G} 0$ 326 group with respect to those identified in G1) was as follows: 1.6 in the oestrogen 327 metabolism pathway; 2.0 in the irinotecan pathway; 1.5 in oxidative stress, and 1.4 in 328 the TGF Receptor Signalling Pathway. Enrichment analyses for the Gene Ontology biological process and molecular 330 function are represented in Fig. 5. The biological processes that proved to be 331 significantly enriched in the gallbladder were all metabolic processes. The most 332 outstanding differences between $\mathrm{G} 0$ and $\mathrm{G} 1$ were: (i) the higher number of proteins 333 involved in the carbohydrate and monosaccharide metabolic processes identified in the 334 infected mice (G1) and, (ii) the higher number of proteins involved in coenzyme and 
335 cofactor metabolic processes identified in the G0 group with respect to the G1 mice

336 (Fig. 5).

337

Regarding molecular functions, the group of proteins significantly enriched in

338 the samples analyzed were those with a binding function and catalytic activity. As can

339 be seen in Fig. 5, the most salient result was the greater number of genes with

340 glutathione transferase activity present in the gallbladders of the G0 group as compared

341 with G1. Overall, we identified up to 19 members of different classes (alpha, mu, kappa,

342 pi and theta) of the glutathione S-transferase enzyme family, 15 in the gallbladders of

343 infected mice and up to 19 in the gallbladders of non-infected mice (see Supplementary

344 Table 1).

345

346

4. DISCUSSION

347

348

As stated in the introduction section, bile is a body fluid produced by the liver

349 that has digestive and excretion functions and its formation depends on the structural

350 and functional integrity of the bile-secretory apparatus $[16,17]$. The main components

351 of bile are bile salts, fatty acids and cholesterol, corresponding to a protein content of

352 only $7 \%$. The sources of proteins in bile include plasma, hepatocytes and the epithelium

353 of the biliary tract and the gallbladder [17]

354

Regarding schistosomiasis, there is in fact no evidence indicating that

355 schistosome infection elicits any kind of biliary disease. Indeed, only a few isolated

356 cases of patients with schistosomal cholecystitis and associated with concomitant

357 gallstones have been reported (less than 10 cases have been described in the medical

358 literature) [18-21]. Nevertheless, considering that schistosome infections cause

359 important liver lesions that also affect the bile secretory apparatus, it is highly likely that 
biliary secretion would be affected in infected animals. Moreover, since some biliary

361 proteins are produced by hepatocytes and the epithelium of the biliary tree, it could be

362

363

364

365

366

367

368

369

370

371

372

373

374 surmised that such infections might also modify the bile protein content. Accordingly, and bearing in mind that there is little information about this aspect either, we were prompted to determine whether any kind of change in the proteome of the gallbladder might arise in mice infected with S. bovis for 4 months.

Regarding the composition of bile, although the chemical composition of human bile is well understood it is only recently when studies have begun to unravel its protein constituents. In the last 10 years, in parallel with the development of proteomic techniques, studies addressing human bile proteins have advanced considerably and we now have available a comprehensive catalogue of biliary proteins [11]. The aims of these studies have been to improve the understanding of the physiological processes involved in the regulation of the hepato-biliary system; to explore the involvement of bile proteins in health and disease, and to discover biomarkers for biliary tract malignancies $[10,11,17,22-24]$. Such work has also served to optimize protocols for sample preparation and to solve the pitfalls involved in the proteomic analysis of bile samples that result from the massive concentration of interfering compounds [17].

In the present work we used a protocol to study the proteins present in bile samples similar to that described by Farina et al. [10], based on sample fractionation by serial centrifugation and acrylamide gel electrophoresis. The aim of this was to reduce the complexity of the bile samples before mass spectrometry and to limit unavoidable losses related to other additional purification methods [17]. In this study with S. bovis we analyzed complete gallbladders instead of bile samples, as has been the case in other studies addressing the human bile proteome, owing to the difficulty involved in collecting bile samples from mice in sufficient quantity without being contaminated 
385

386

387

388

389

390

391

392

393

394

395

396

397

398

399

400

401

402

403

404

405

406

407 biological pathways and relevant molecular functions were underrepresented in the

408

409

with tissue proteins. This problem was exacerbated in the group of mice infected with $S$. bovis, having these mice an apparent reduction in gallbladder volume (not shown), effect that has already been reported in patients with portal hypertension due to advanced Schistosoma mansoni infection [17].

After processing the gallbladders of the infected and non-infected mice, the small differences observed in the profile of the protein fraction bands (Fig. 1) afforded the first indications of possible modifications in the biliary proteome due to S. bovis infection. The analysis by LCAS/MS of these fractions revealed 1589 and 1489 nonredundant proteins in mice not infected and infected with S. bovis respectively. Bearing in mind that we analyzed complete gallbladders, this number is compatible with the 812 proteins identified by Farina et al. [11] in a similar proteomic analysis of human bile. Initially, the comparison of the biliary proteomes from non-infected and infected mice did not revealed important differences in either the number or the type of proteins identified, as inferred from the "Protein class classification" (see Fig 2). However, after a more detailed analysis of some groups of proteins, such as those included in the oxidoreductase class, together with the analysis of the enrichment of the wikipathways and Gene Ontology categories, we did detect significant differences between the mice infected and not infected with S. bovis. It should be noted that some of the differences observed in the protein composition between the $G 0$ and $\mathrm{Gl}$ groups could in fact represent quantitative differences in the expression level of the proteins, since the least abundant proteins would be below the threshold of detection by MS.

The analysis and comparison of the respective proteomes revealed that several biliary proteome of the infected mice ( $\mathrm{G} 1$ group). Thus, in the gallbladders of the infected mice we observed the expression of a significantly lower number of proteins 
410 involved in several pathways, being especially relevant among them the irinotecan 411 pathway, oestrogen metabolism and oxidative stress. We also observed a lower number 412 of components with metabolic functions and of molecules with glutathione S413 transferase activity (see Fig. 4 and 5). Irinotecan (IRI) is an anti-proliferative cytotoxic 414 agent used to treat metastatic colorectal cancer that is metabolized in the liver and 415 converted to SN-38, the active metabolite [26]. Additionally, the liver -and other 416 peripheral tissues-is a site for the biosynthesis of oestrogens and it is also the main site 417 for further biotransformation [27]. Three of the hepatic biological processes that are 418 affected by S. bovis infection -namely irinotecan metabolism, the biosynthesis and 419 biotransformation of oestrogens and oxidative stress-involve several components of the 420 Cytochrome P450 family (CYP), glutathione Stransferase (GST) and the UDP 421 glucuronyltransferase family (UGT). Hepatic CYP, GST and UGT are three multigene families of enzymes that play a

423 critical role in the metabolism of many drugs and xenobiotics [28-30]. It has been 424 reported that the activities and levels of expression of these enzymes are altered by 425 inflammatory stimuli, including helminthic infections [31]. In this sense, it has been 426 shown that in chronic S. mansoni infection (late phase of schistosomiasis) the liver 427 content and activity of some isoforms of CYP, GST and UDP are downregulated and 428 these regulations seem to be associated with a greater induction of Th2 cytokines [32429 35]. The biliary proteome of the mice infected with S. bovis studied in the present work 430 reflects a situation similar to that described for the liver, where the numbers of CYP, 431 GST and UGT isoforms are underrepresented with respect to normal values (16 CYP, 43215 GST and 12 UGT isoforms in gallbladder in mice infected with S. bovis; 39 CYP, 19 433 GST and 19 UGT isoforms in gallbladder from non-infected mice) (see Supplementary 434 Table 1). Our results corroborate those reported by other authors for the liver and show 
435 that the elimination of drugs via both CYP and phase II of drug metabolism such as

436 GST and UGT may be severely impaired during murine chronic schistosomiasis [35]. In

437 fact, it has been demonstrated that chronic S. mansoni infection influence the 438 pharmacokinetic profiles of some drugs, such as praziquantel, enpiroline and 439 mefloquine, leading to delayed clearance [36, 37]. Accordingly, given that 440 schistosomiasis is prevalent in countries where coinfection for diseases such as HIV or 441 malaria is common, it is important to ask how schistosomiasis affects the enzymes 442 responsible for the metabolism of drugs given to treat not only schistosomiasis but also 443 these coinfections [35].

$444 \quad$ Regarding the signalling molecules and pathways significantly enriched in the 445 G1 group of infected animals (Fig.4), several observations deserve further comment, 446 including: (i) the upregulation of pathways involved in chronic inflammation induction 447 such as the EGFRI and the $\mid \mathrm{L}-6$ signalling pathways, which are cross-connected and 448 have important roles in the proliferation of key inflammatory hepatic cells. [38]; (ii) the 449 upregulation of these two ways could in turn be related to the increase in the FAS450 mediated apoptotic pathway also observed in our study, since inflammation is targeted 451 once activated the apoptotic cascade [39]; (iii) the increase in the cytokine IL-6 can also 452 be related to lipid metabolism disorders, in order to modulate them accordingly [40] and 453 (iv) concerning the observed increase in the T-cell receptor signalling pathway, it is well 454 established that schistosome infection promotes T-cell activation and a Th2-type 455 inflammatory pathology, which requires host T-cell receptor signals, favouring parasite 456 growth and development in the mammalian host [41, 42]. Then, the upregulation of this 457 signalling pathway in the gallbladder -and liver-would favour the Th2-type cells that 458 are critical during helminthic infections and support the deep effects of the parasite459 induced inflammatory environment on the host. 
461 gallbladder and provides a comprehensive catalogue of biliary proteins that may be of 462 great use in other studies addressing biliary physiology and pathology. We show that 463 chronic schistosome infections cause significant changes in the biliary proteome that 464 may produce physiological alterations and affect the therapeutic actions of drugs when 465 administered to human patients and animals with schistosomiasis.

466 
474 
481 [4] Andrade ZA. Schistosomal Hepatopathy. Mem Inst Oswaldo Cruz 2004;99:51-7.

482 [5] Andrade ZA. Schistosomiasis and liver fibrosis. Parasite Immunol 2009;31:656-33.

483 [6] Harvie M, Jordan TW, La Flamme AC. Differential liver protein expression during 484 schistosomiasis._Infect Immun 2007;75:736-44.

485 [7] Manivannan B, Jordan TW, Secor WE, La Flamme AC. Proteomic changes at 8 486 weeks after infection are associated with chronic liver pathology in experimental 487 schistosomiasis. J Proteomics 2012;75:1838-48.

488 [8] Oliveira L, Andrade ZA. Significance of bile-duct changes in schistosomiasis. Rev 489 Soc Bras Med Trop 2005;38:464-68.

490 [9] Loebermann M, Sombetzki M, Langner C, Fuchsbichler A, Gumhold J, Silbert D, 491 Riebold D, Holtfreter M, Fickert P, Nizze H, Trauner M, Reisinger EC. Imbalance of 492 pro- and antifibrogenic genes and bile duct injury in murine Schistosoma mansoni 493 infection-induced liver fibrosis. Trop Med Int Health 2009;14:1418-25.

494 [10] Farina A, Dumonceau JM, Delhaye M, Frossard JL, Hadengue A, Hochstrasser 495 DF, Lescuyer P. A step further in the analysis of human bile proteome. J Proteome Res $496 \quad 2011 ; 10: 2047-63$.

497 [11] Farina A, Delhaye M, Dumonceau JM. Bile proteome in health and disease. 498 Compr Physiol 2014;4:91-108. 
499 [12] Laemmli UK. Cleavage of structural proteins during the assembly of the head of

500 bacteriophage T4. Nature 1970; 227: 680-85.

501 [13] Mi H, Muruganujan A, Thomas PD. PANTHER in 2013: modeling the evolution

502 of gene function, and other gene attributes, in the context of phylogenetic trees. Nucleic

503 Acids Res 2013;41(Database issue):D377-86.

504 [14] Duncan DT, Prodduturi N, Zhang B. WebGestalt2: an updated and expanded

505 version of the Web-based Gene Set Analysis Toolkit. BMC Bioinformatics

506 2010;11(Supp| 4):P10.

507 [15] Benjamini $Y$, Hochberg $Y$. Controlling the false discovery rate: a practical and

508 powerful approach to multiple testing. J R Statist Soc B 1995;57: 289-300.

509 [16] Esteller A. Physiology of bile secretion. World J Gastroenterol 2008;14:5641-49.

510 [17] Farina A, Dumonceau JM, Lescuyer P. Proteomic analysis of human bile and

511 potential applications for cancer diagnosis. Expert Rev Proteomics 2009;6:285-301.

512 [18] Rappaport I, Albukerk J, Schneider IJ. Schistosomal cholecystitis. Arch Pathol

$513 \quad 1975 ; 99: 227-28$.

514 [19] al-Saleem T, al-Janabi T. Schistosomal cholecystitis: report of six cases. Ann R

515 Coll Surg Engl 1989;71:366-367.

516 [20] el Shiekh Mohamed AR, al Karawi MA, Yasawy Ml. Organ involvement in

517 hepato-intestinal schistosomiasis. Hepatogastroenterology 1994;41:370-76.

518 [21] Manes K, Chatzimargaritis K, Apessou D, Papastergiou V, Dervenis C.

519 Granulomatous cholecystitis in a patient with Schistosoma mansoni infection: A case 520 report. |JCRI 2014;5:439-43.

521 [22] Kristiansen TZ, Maitra A, Pandey A. Proteomics of human bile. In Proteomics of 522 human body fluids. Edited by Visith Thongboonkerd. Humana Press, New Jersey 2007,

523 pp. 399-414 
524 [23] Barbhuiya MA, Sahasrabuddhe NA, Pinto SM, Muthusamy B, Singh TD,

525 Nanjappa V, Keerthikumar S, Delanghe B, Harsha HC, Chaerkady R, Jalaj V, Gupta S,

526 Shrivastav BR, Tiwari PK, Pandey A. Comprehensive proteomic analysis of human

527 bile._Proteomics 2011;11:4443-4453.

528 [24] Farid SG, Craven RA, Peng J, Bonney GK, Perkins DN, Selby PJ, Rajendra Prasad

529 K, Banks RE. Shotgun proteomics of human bile in hilar cholangiocarcinoma.

530 Proteomics 2011;11:2134-2138.

531 [25] Ali QM, Abdel-Rahim IM, Doehring-Schwertfegar E, Franke D, Kardorff R, el

532 Sheikh M, Ehrich $\mathrm{JH}$. Ultrasonographic evaluation of gallbladder function in patients

533 with Schistosoma mansoni infection. Trop Doct 1990;20:113-5.

534 [26] Nagar S, Blanchard RL. Pharmacogenetics of uridine

535 diphosphoglucuronosyltransferase (UGT) 1A family members and its role in patient

536 response to irinotecan. Drug Metab Rev 2006;38:393-409.

537 [27] Jefcoate, C.R., Liehr, J.G., Santen, R.J., Sutter, T.R., Yager, J.D., Yue, W.,

538 Santner, S.J., Tekmal, R., Demers,L., Pauley, R., Naftolin, F., Mor, G., Berstein, L.

539 Tissue-specific synthesis and oxidative metabolism of estrogens. J Natl Cancer Inst

540 Monogr 2000;27:95-112.

541 [28] King CD, Rios GR, Green MD, Tephly TR. UDP-glucuronosyltransferases. Curr

542 Drug Metab 2000;1:143-61.

543 [29] Sherrat PJ, Hayes JD. Glutathione S-transferase. In: loannides C, editor. Enzyme

544 systems that metabolize drugs and other xenobiotics. John Wiley \& Sons Ltd; 2001, p.

545 [30] Estabrook RW: A passion for P450s (rememberances of the early history of

546 research on cytochrome P450)._Drug Metab Dispos 2003, 31:1461-73. 
547 [31] Conte FP, Fidalgo-Neto AA, Manhães-Rocha DA, Paumgartten FJ, De-Oliveira

548 AC. Activity of liver microsomal enzymes during the chronic phase of murine

549 schistosomiasis. Braz J Med Biol Res 2007;40:657-62.

550 [32] Sheweita SA, Mangoura SA, el-Shemi AG. Different levels of Schistosoma

551 mansoni infection induce changes in drug-metabolizing enzymes. J Helminthol

$5521998 ; 72: 71-77$.

553 [33] Sheweita SA, Mubark J, Doenhofe MJ, Mostafa MH, Margison GP, O'Connor PJ,

554 Elder $\mathrm{RH}$. Changes in the expression of cytochrome P450 isozymes and related

555 carcinogen metabolizing enzyme activities in Schistosoma mansoni-infected mice. J

556 Helminthol 2002;76:71-78.

557 [34] Sheweita SA. Drug-metabolizing enzymes: mechanisms and functions. Curr Drug

558 Metab 2000; 1:107-32.

559 [35] Mimche SM, Nyagode BA, Merrell MD, Lee CM, Prasanphanich NS, Cummings

560 RD, Morgan ET. Hepatic cytochrome P450s, phase II enzymes and nuclear receptors

561 are downregulated in a Th2 environment during Schistosoma mansoni infection. Drug

562 Metab Dispos 2014;42:134-40.

563 [36] Gotardo MA, Hyssa JT, Carvalho RS, De-Carvalho RR, Gueiros LS, Siqueira CM,

564 Sarpa M, De-Oliveira AC, Paumgartten Jr F. Modulation of expression and activity of

565 cytochrome P450s and alteration of praziquantel kinetics during murine

566 schistosomiasis. Mem Inst Oswaldo Cruz. 2011; 106:212-9.

567 [37] Ingram K, Duthaler U, Vargas M, Ellis W, Keiser J. Disposition of mefloquine and

568 enpiroline is highly influenced by a chronic Schistosoma mansoni infection. Antimicrob

569 Agents Chemother. 2013; 57:4506-11

570 [38] Lanaya H, Natarajan A, Komposch K, Li L, Amberg N, Chen L, Wculek SK,

571 Hammer M, Zenz R, Peck-Radosavljevic M, Sieghart W, Trauner M, Wang H, Sibilia 
572 M. EGFR has a tumour-promoting role in liver macrophages during hepatocellular

573 carcinoma formation. Nat Cell Biol. 2014; doi: 10.1038/ncb3031.

574 [39] Schneider-Brachert W, Heigl U, Ehrenschwender M. Membrane trafficking of

575 death receptors: implications on signalling. Int J Mol Sci 2013;14:14475-503.

576 [40] Hassan W, Ding L, Gao RY, Liu J, Shang J. Interleukin-6 signal transduction and

577 its role in hepatic lipid metabolic disorders. Cytokine 2014;66:133-42.

578 [41] Xu X1, Wen X, Chi Y, He L, Zhou S, Wang X, Zhao J, Liu F, Su C. Activation579 induced $T$ helper cell death contributes to Th1/Th2 polarization following murine 580 Schistosoma japonicum infection. J Biomed Biotechnol 2010;2010:202-397

581 [42] Tang $H$, Ming Z, Liu R, Xiong T, Grevelding CG, Dong H, Jiang M. Development 582 of adult worms and granulomatous pathology are collectively regulated by $T$ - and B583 cells in mice infected with Schistosoma japonicum. PLoS One 2013;8:e54432. 
586 Figure captions

587

588 Figure 1. Silver-stained $10 \%$ polyacrylamide gels showing the protein fractions

589 obtained from gallbladder homogenates of non-infected (G0) and Schistosoma bovis-

590 infected mice $(\mathrm{G} 1)$. Gel lanes were sliced into 3 pieces and the resulting gel slices were

591 digested with trypsin and analyzed by LC-MS/MS. S-1 and P-1, supernatant and pellet

592 from gallbladder homogenate centrifugation at 17,000g for $30 \mathrm{~min}$. S-2 and P-2,

593 supernatant and pellet from centrifugation of S-1 at 100,000g for $1 \mathrm{~h}$.

594

595 Figure 2. The proteins identified in the gallbladder from non-infected mice (G0) and 596 mice infected with Schistosoma bovis (G0) were classified in "Protein classes" using the

597 Panther Classification System. Bars represent the percentage of proteins in each protein 598 class relative to the total number of proteins in the group.

599

600 Figure 3. Classification according to their molecular function of the proteins identified 601 only in non-infected mice (G0), only in mice infected with Schistosoma bovis (G1) and 602 in both groups of mice ( $G 0$ and $G 1$ ). Pie charts represent the percentages of proteins in 603 each category without including the proteins with catalytic activity.

604

605 Figure 4. Ratio of enrichment (R) of the 48 significantly enriched pathways associated 606 with proteins identified in the proteome of the gallbladders of non-infected (G0) and 607 Schistosoma bovis-infected (G1) mice. Significance criteria included $p<0.0001$ and a 608 minimum number of 6 genes for each category.

609 
610 Figure 5. Ratio of enrichment $(R)$ of top 10 enriched biological processes and molecular

611 functions associated with proteins identified in the proteome of the gallbladders of non-

612 infected (G0) and Schistosoma bovis-infected mice (G1). Significance criteria included

$613 p<0.0001$ and a minimum number of 6 genes for each category.

614 
Table 1. Number of unique proteins identified in the fractions of the gallbladder homogenates. Redundant identifications, keratins and immunoglobulins have been excluded. G0, non-infected mice; G1, mice infected with Schistosoma bovis.

\begin{tabular}{|c|c|c|c|}
\hline Gallbladder fractions & Go & & G1 \\
\hline S-1 (17,000g supernatant) & 998 & & 1069 \\
\hline P-1 (17,000g pellet) & 768 & & 752 \\
\hline S-2 (10 $\mathrm{g}$ supernatant $)$ & 300 & & 379 \\
\hline \multirow[t]{2}{*}{ P-2 $\left(10^{b} \mathrm{~g}\right.$ pellet $)$} & 1044 & & 757 \\
\hline & 1589 & & 1489 \\
\hline Specific and shared & Only G0 & G0 and G1 & Only G1 \\
\hline identifications & 448 & 1141 & 348 \\
\hline
\end{tabular}


Table 2. Proteins identified in the gallbladder of Schistosoma bovis infected (G1) and non-infected (G0) biological pathways: Estrogen metabolism, Irinotecan pathway, Oxidative Stress and TGF-beta Receptor Signali

\begin{tabular}{|c|c|c|c|c|c|}
\hline $\begin{array}{c}\text { Experimental } \\
\text { group }\end{array}$ & Uniprot ID & $\begin{array}{l}\text { Gene } \\
\text { ID }\end{array}$ & Gene name & Protein name & Pathway \\
\hline GO & P13745 & 14857 & Gstal & glutathione S-transferase, alpha $1(Y a)$ & Estrogen metab \\
\hline G0 & P00184 & 13076 & Cyplal & cytochrome P450, family 1 , subfamily a, polypeptide 1 & Estrogen metabo \\
\hline G0 & Q8VCT4 & 104158 & Ces3 & carboxylesterase 3 & Irinotecan pathw \\
\hline G0 & Q5FW80 & 394430 & Ugtla10 & UDP glycosyltransferase 1 family, polypeptide A10 & Irinotecan pathw \\
\hline G0 & P70691 & 22236 & Ugtla2 & UDP glucuronosyltransferase 1 family, polypeptide A2 & Irinotecan pathw \\
\hline G0 & Q8R0P3 & 394435 & Ugtla6b & UDP glucuronosyltransferase 1 family, polypeptide A6B & Irinotecan pathw \\
\hline G0 & Q61133 & 14872 & Gstt2 & glutathione S-transferase, theta 2 & Oxidative Stress \\
\hline G0 & P09671 & 20656 & Sod2 & superoxide dismutase 2, mitochondrial & Oxidative Stress \\
\hline G0 & P28652 & 12323 & Camk2b & calcium/calmodulin-dependent protein kinase II, beta & TGF-beta Recept \\
\hline G0 & Q8K1M3 & 19087 & Prkar2a & protein kinase, cAMP dependent regulatory, type II alpha & TGF-beta Recept \\
\hline G0 & P07214 & 20692 & Sparc & secreted acidic cysteine rich glycoprotein & TGF-beta Recept \\
\hline G0 & Q6P5F9 & 103573 & Xpol & exportin 1, CRM1 homolog (yeast) & TGF-beta Recept \\
\hline G1 & Q64669 & 18104 & Nqo 1 & $\mathrm{NAD}(\mathrm{P}) \mathrm{H}$ dehydrogenase, quinone 1 & Estrogen metabo \\
\hline G0, G1 & $\mathrm{B} 6 \mathrm{VGH} 4$ & 13077 & Cypla2 & cytochrome P450, family 1, subfamily a, polypeptide 2 & Estrogen metabo \\
\hline G0, G1 & P10649 & 14862 & Gstm 1 & glutathione S-transferase, mu 1 & Estrogen metabo \\
\hline G0, G1 & Q6XL48 & 394433 & Ugtla5 & UDP glucuronosyltransferase 1 family, polypeptide A5 & Irinotecan pathw \\
\hline G0, G1 & Q6ZQM8 & 394432 & Ugtla7c & UDP glucuronosyltransferase 1 family, polypeptide A7C & Irinotecan pathv \\
\hline G0, G1 & Q62452 & 394434 & Ugtla9 & UDP glucuronosyltransferase 1 family, polypeptide A9 & Irinotecan pathv \\
\hline G0, G1 & Q64435 & 94284 & Ugtla6a & UDP glucuronosyltransferase 1 family, polypeptide A6A & Irinotecan pathw \\
\hline G0, G1 & P97494 & 14629 & Gclc & glutamate-cysteine ligase, catalytic subunit & Oxidative Stress \\
\hline G0, G1 & P11352 & 14775 & Gpx1 & glutathione peroxidase 1 & Oxidative Stress \\
\hline G0, G1 & P47791 & 14782 & Gsr & glutathione reductase & Oxidative Stress \\
\hline G0, G1 & Q9JMH6 & 50493 & Txnrd1 & thioredoxin reductase 1 & Oxidative Stress \\
\hline
\end{tabular}




\begin{tabular}{|c|c|c|c|c|}
\hline G0, G1 & Q9JLT4 & 26462 & Txnrd2 & thioredoxin reductase 2 \\
\hline G0, G1 & Q6PHZ2 & 108058 & Camk2d & calcium/calmodulin-dependent protein kinase II, delta \\
\hline G0, G1 & Q9QZD9 & 54709 & Eif3i & eukaryotic translation initiation factor 3, subunit I \\
\hline G0, G1 & P47811 & 26416 & Mapk14 & MAP kinase 14 \\
\hline G0, G1 & P49817 & 12389 & Cavl & caveolin 1, caveolae protein \\
\hline G0, G1 & Q02248 & 12387 & Ctnnb 1 & catenin (cadherin associated protein), beta 1 \\
\hline G0, G1 & P63017 & 15481 & Hspa8 & heat shock protein 8 \\
\hline G0, G1 & P70168 & 16211 & Kpnb 1 & karyopherin (importin) beta 1 \\
\hline G0, G1 & Q9Z1Z2 & 20901 & Strap & serine/threonine kinase receptor associated protein \\
\hline G0, G1 & Q922Z3 & 68015 & Trapl & TNF receptor-associated protein 1 \\
\hline
\end{tabular}

Oxidative Stress TGF-beta Recept TGF-beta Recept TGF-beta Recept TGF-beta Recep TGF-beta Recept TGF-beta Recep TGF-beta Recept TGF-beta Recept TGF-beta Recept 


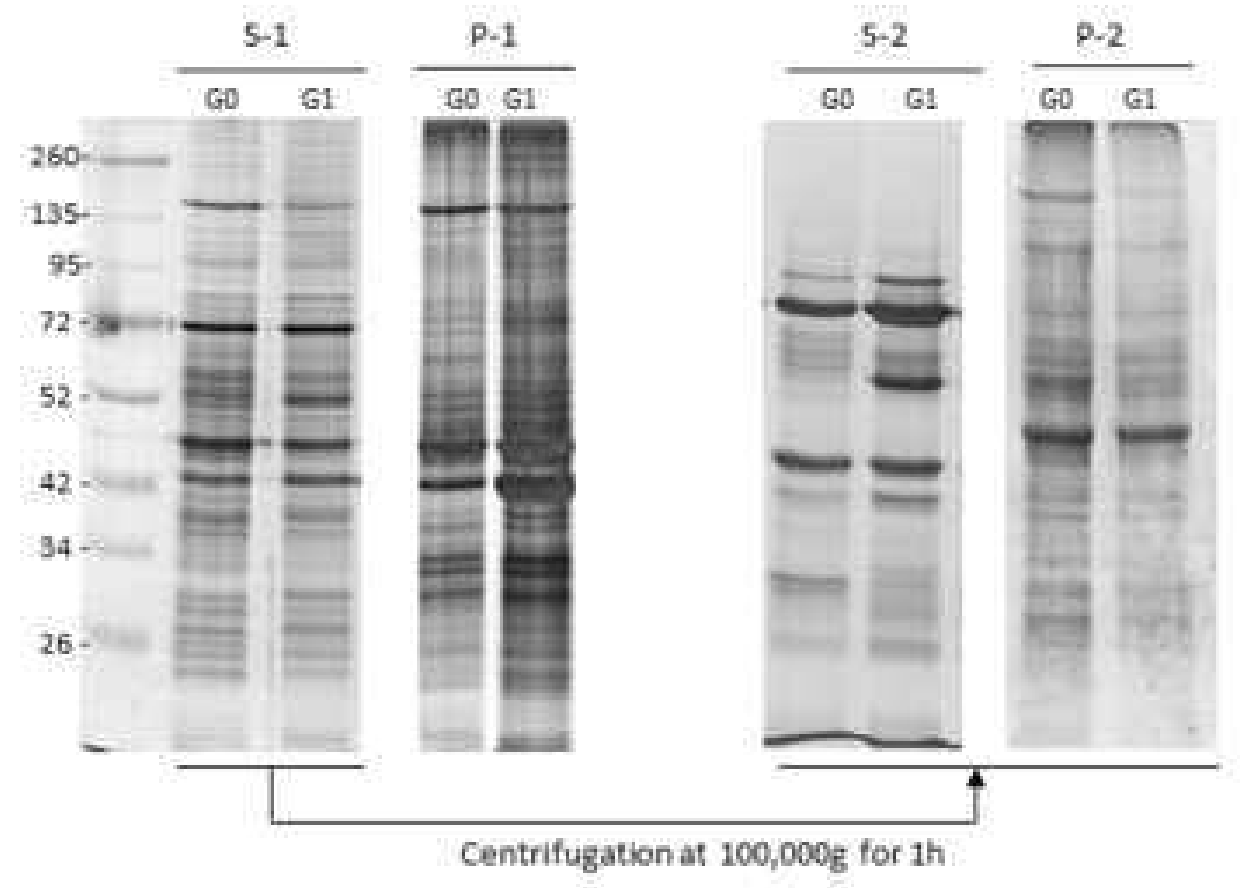

Figure 1

Figure 1

Figre 1

Centrifugation at $100,000 \mathrm{~g}$ for $1 \mathrm{~h}$.

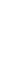

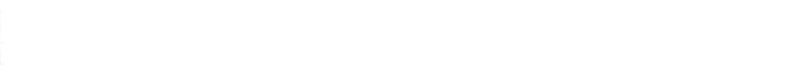


Figure 2

Figure 2

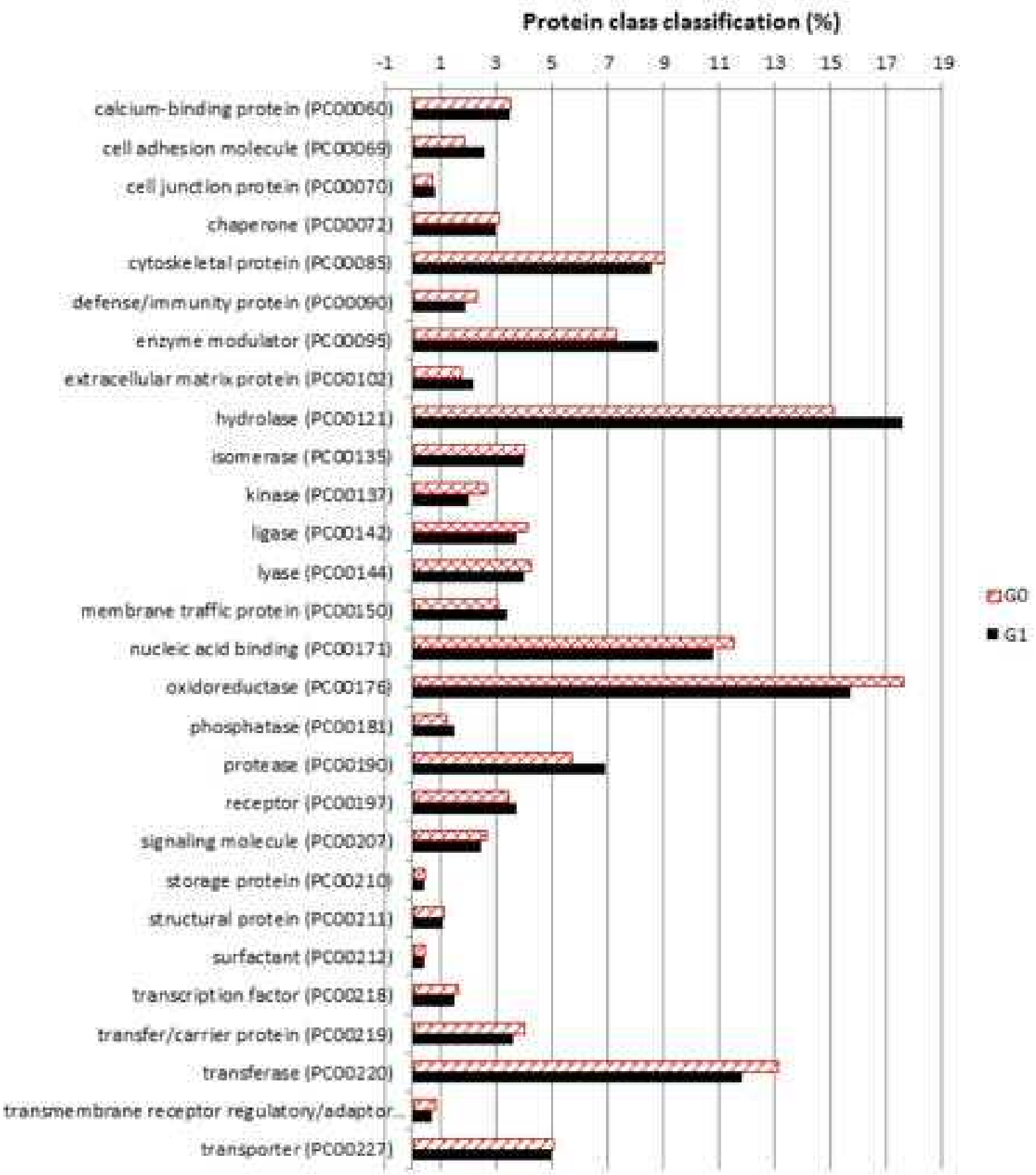

960 
Figure 3
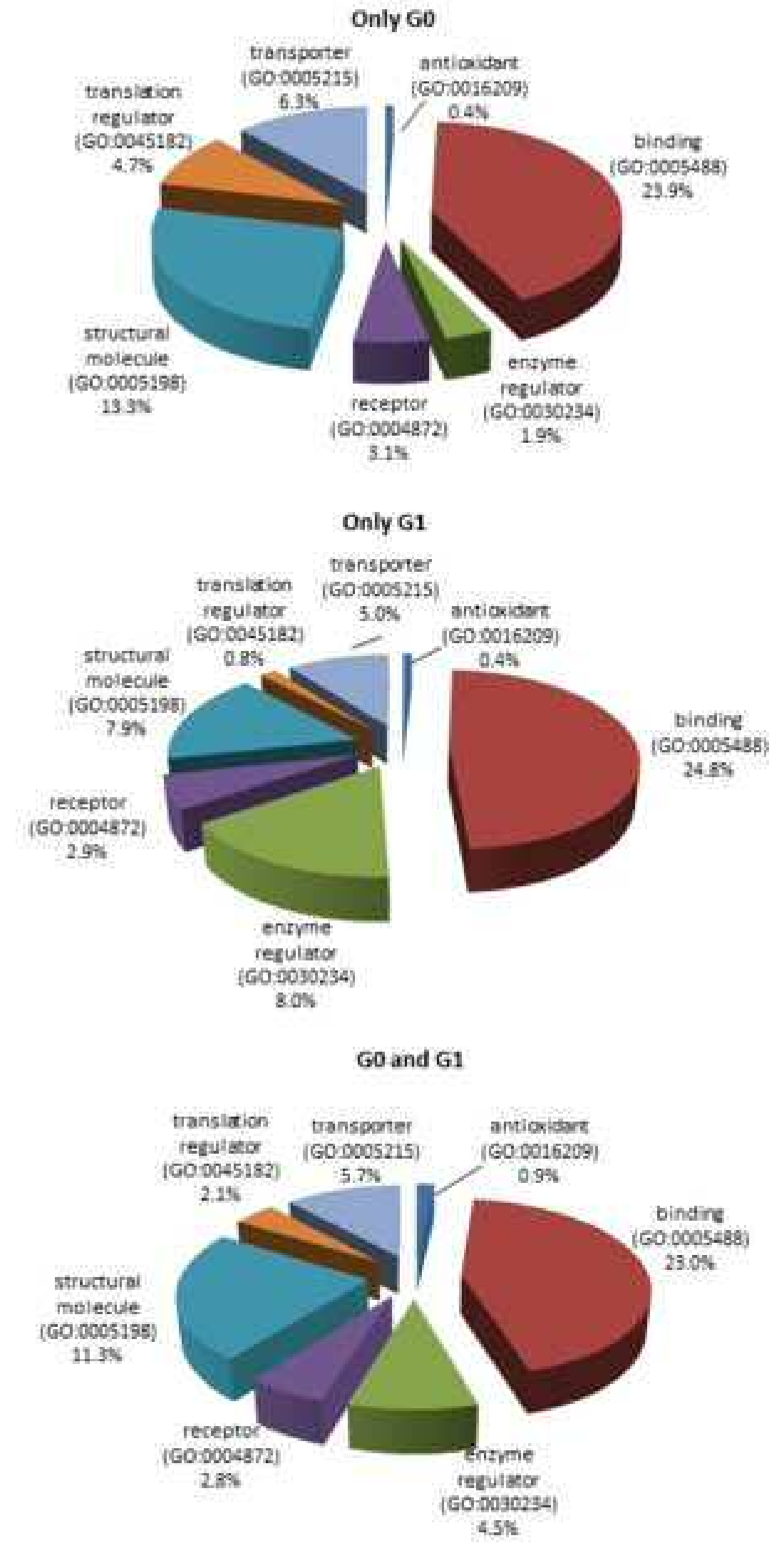

Figure 
Figure 4

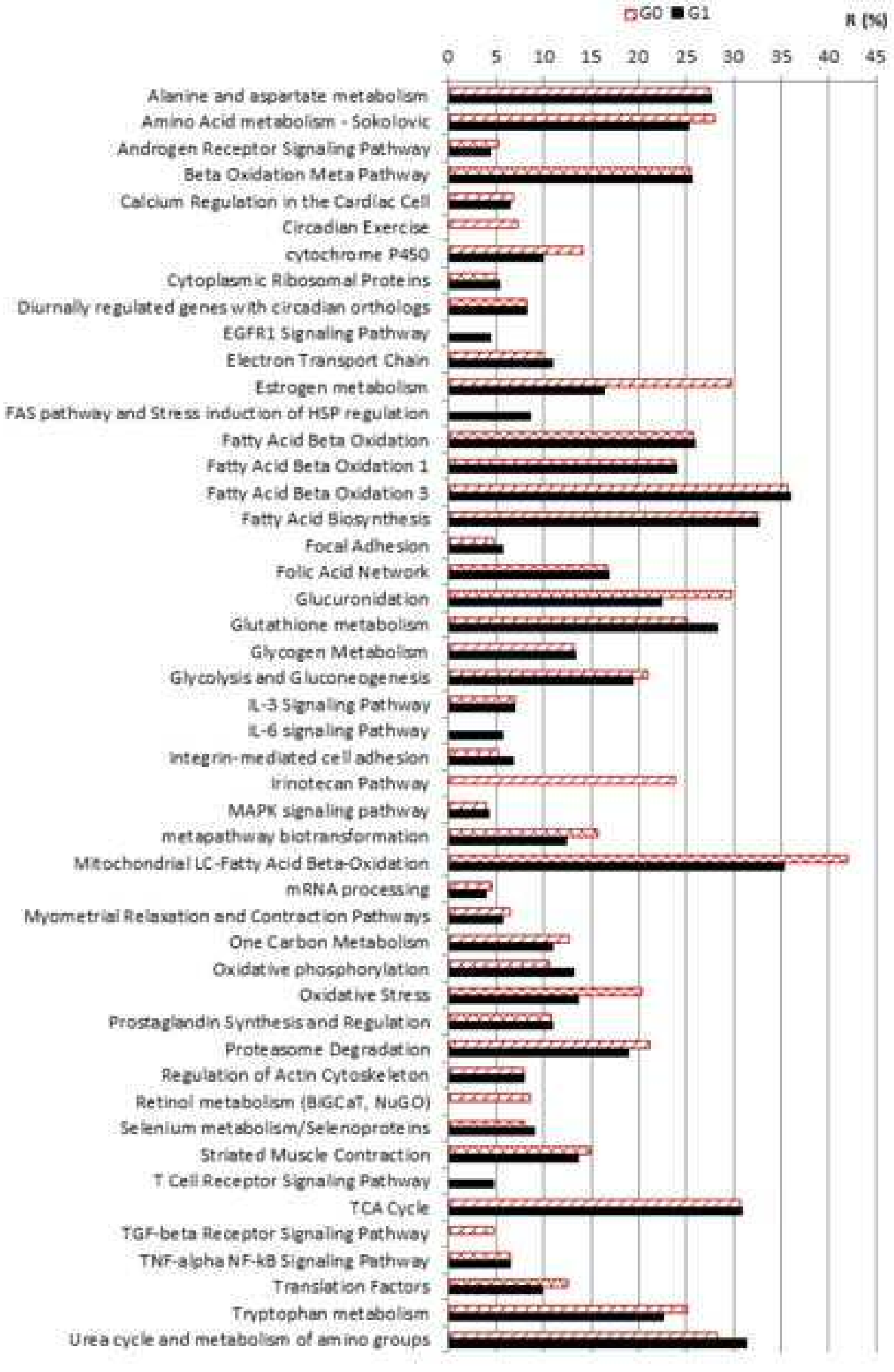

Urea cycle and metabolism of am ino groups

Amino Acid metabolism - Sololow
Androgen Receptor Siznaling Pathwe
Beta Oxidation Meta Pathwe
Calcium Regulation in the Cardlac Ce
Circadien Exercis
cytochrome p45
Crtoplasmic Ribosamal Prote in ctron Transoort Chain ction of HSP regulation

Peid Beta Oudation 3

Focald athesion

Glucuronidation

tutathione metabolism

Glycogen Metabolign

medirted celli adhesion

irinotecan Pathwo

MAPK signaline pathway

met aosthwav biotransformatian

Contract ion Pathways

One Carbon Metabolism

Dridetive stien I

Prostaglanid in $5 y$ inthesis and Regulat on

Proteasome Degradotion

Rezulation of Actin Cytoskeleton

Retinol metabolsm \{aigC,ai, NuGO

Selenium metibolism/Selenoprote ins

Striated Muscle Contraction

TCA Cycle

GF-beta tieceptor Siznaling Pathway

Signaline Pathwe

Transfotion Factors

rvptophan metebolism

\footnotetext{
政
} 
Figure 5

\section{Biological process}

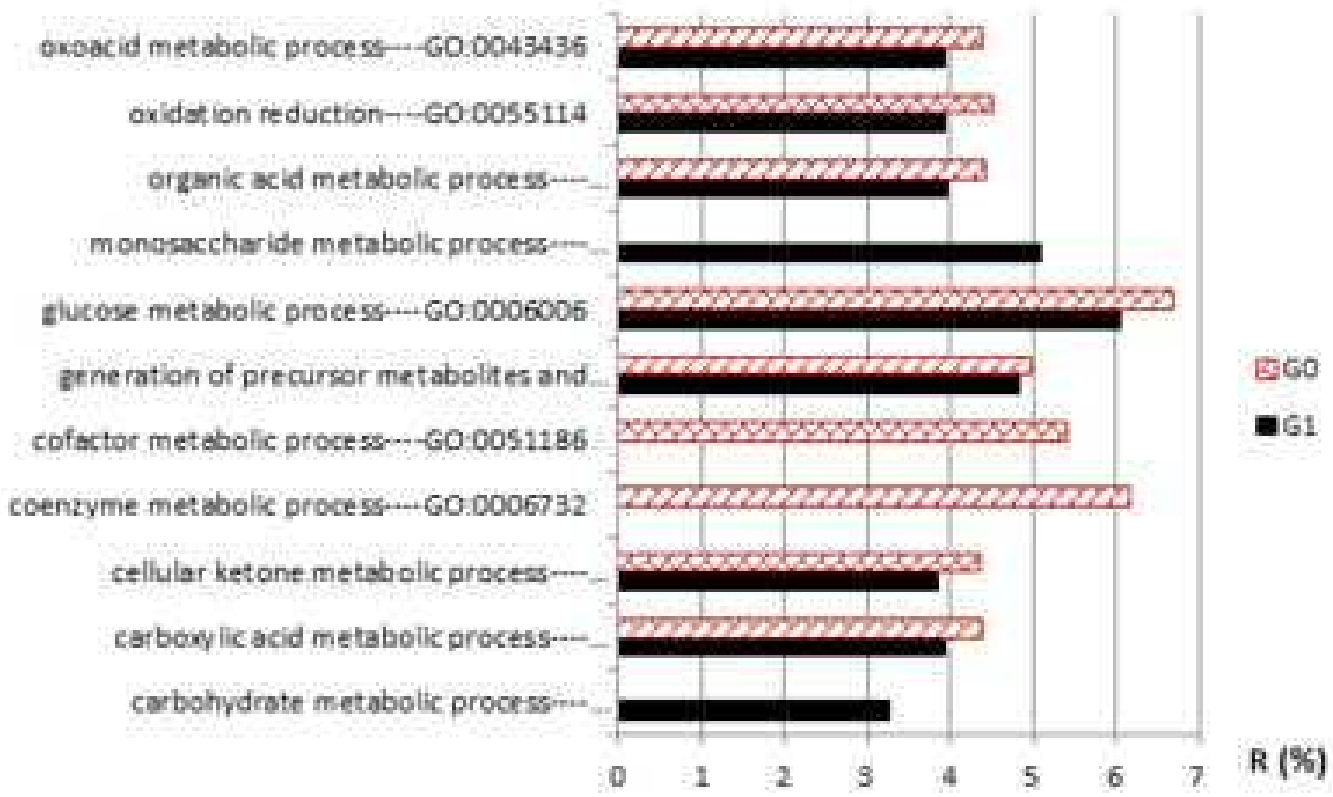

\section{Molecular function}

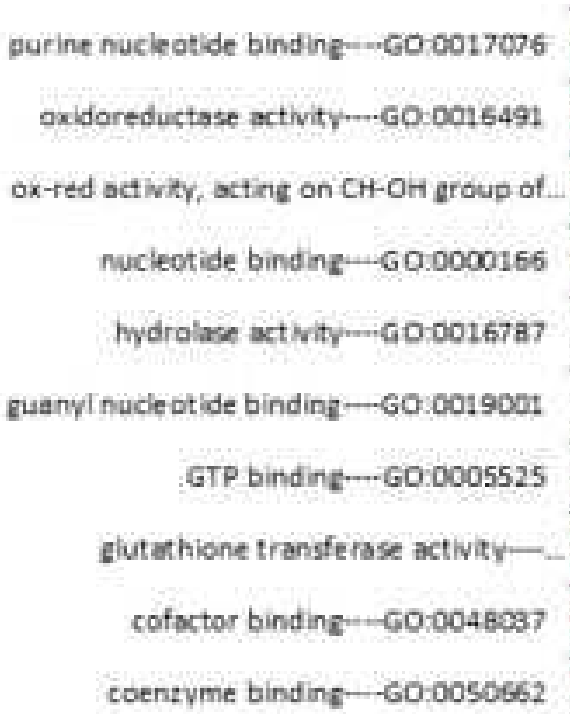

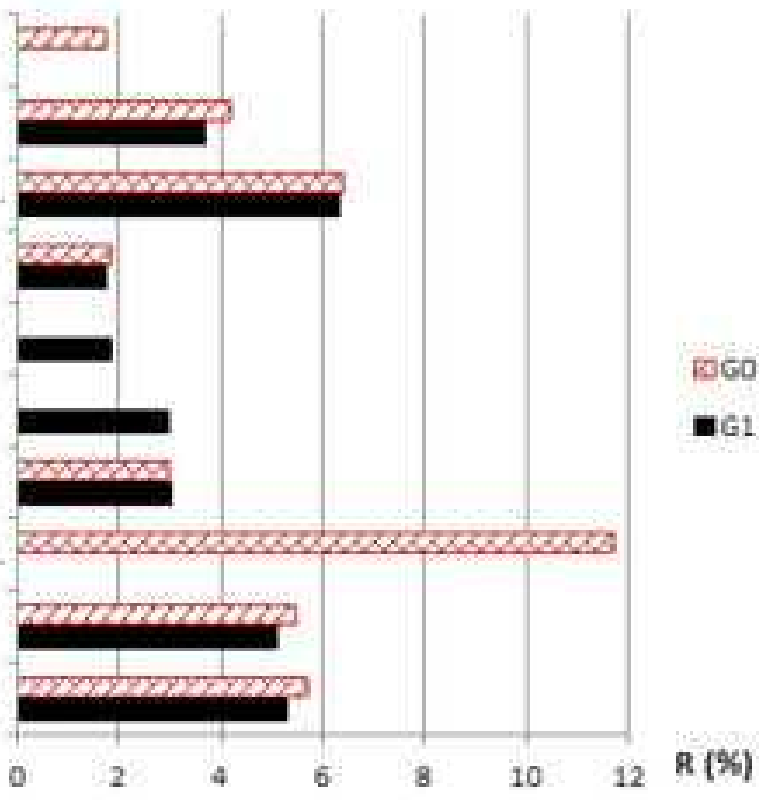

